\title{
The Production and Application of digital narrative in ESL Classroom
}

\author{
Yu Zhou ${ }^{1}$, Xue-xin Cui ${ }^{2}$ \\ ${ }^{1)}$ School of Qiu Zhen, Huzhou University, Zhejiang, China \\ 2) School of Qiu Zhen, Huzhou University, Zhejiang, China
}

\begin{abstract}
ESL digital narratives are defined as 2-10 minutes digital telling stories which combine images or videos with narrations, sound effects or music. This article will focus on the production and application of digital narratives for ESL classroom. With digital narrative teaching model, ESL students are offered the opportunities to intake "the comprehensible input", cultivate the literacy development and improve their language skills in reading, listening, speaking and writing.
\end{abstract}

Keywords - digital narrative, ESL teaching, design principles

\section{英语教学“数字叙事”制作与运用}

\author{
周昱 崔学新 \\ 湖州师范学院求真学院, 湖州, 浙江, 中国
}

摘 要 随着科技的飞速发展, 数字设备被广泛运用于教育领域来辅助教学活动, 数字化讲述便是其中之一。它通常以 2-10 分钟的 “数字叙事”来传达概念、展现主题、表现感情色彩。基于“数字叙事”的课堂教学有助于提高知识的摄入量, 培养学习者的创新思维与 批判精神。本文将探讨“数字叙事”的简单制作过程与课堂应用实例。将“数字叙事”模式融入英语教学有助于提供“有意义的语言输入”, 促成文化素养的生成, 支持语言理解能力的纵深发展。

关键词 数字叙事; 外语教学; 设计理念

\section{1. 引言}

故事讲述作为一种传播知识、智慧、价值的方式一直 被沿用至今。数字叙事是信息时代对故事讲述这一古老艺 术形式的全新阐释。它借助多媒体工具来传达概念, 展现 主题和某种特定的感情色彩[1]。随着科技的普及, “数字叙 事”开始走进课堂, 并成为一种颇受欢迎的教学辅助手段。

\section{2. “数字叙事”模式所反映的教学思想}

以教学为目标的 “数字叙事”反映了认知主义的学习 论。认知主义认为: 学习是主动地形成类目及其编码系统 的过程; 信息编码不仅有助于知识的理解, 而且有助于信 息的储存和提取。“数字叙事”借助多媒体进行表语义编码, 有助于引起学习者的注意, 告知学习目标, 提示先前知识,
呈现教材, 引出作业, 提供反馈评估, 促进知识的迁移。

“数字叙事”作为一种教学辅助手段也反映了行为主义 的学习理念。行为主义的“程序教学之父”斯金纳认为: 机 体接受外界的刺激, 做出相关反映, 就是所谓的学习; 在 学习过程中, “教学机器”的使用能够帮助实现正强化刺激。 作为辅助教学的“教学机器”, “数字叙事”的课堂运用遵循着 行为主义固有的原则、步骤和模式。它注重知识信息的分 层展现、强化练习, 能够有效地激发学生的学习兴趣和参 与意识。

运用多媒体数字技术, “数字叙事”能够有效地模拟各 种教学环境, 帮助学习者完成意义的构建。其学习过程体 现了构建主义的主动性、社会性和情景性。小电影的故事 讲述要求教学围绕当前学习主题, 按“最领近发展区”的要 
求建立概念框架, 将学生引入一定的问题情景, 要求学生 独立分析探索, 在概念框架中继续攀升[2]。通过小组协商、 讨论, 学习者的目标知识体系逐渐变得明朗, 在集体思维 的基础上最终完成对所学知识的意义构建。

\section{3. 以英语教学为目标的“数字叙事”制作}

制作一段 1-4 分钟以英语教学为目标的“数字叙事”, 首 先需要创作一段大约四百字的故事脚本, 并将其用自己的 声音录制成音频资料; 然后将音频配以图片资料, 并增添 音乐因素以烘托主要感情基调。

\section{1 创作故事脚本和故事板}

脚本创作即确定话题以及故事类型。例如, 一个关于 环境的话题既可以设计成叙述类的故事, 也可以设计成论 述类的故事; 既可以讲述个人的经历, 也可以表达专业的 学术观点。然后针对所选择的话题进行研究, 搜寻相关原 版资料, 创作出一段 400 字左右的文字, 能够清晰展现出 作者的观点、主线问题、主题感情色彩等因素。以英语教 学为目标的 “数字叙事”脚本制作在语言、语体、内容的选 择上还应符合观众的语言程度, 体现教学目标中的语言重 点和难点。

故事板的制作则是视觉化课本信息的第一步。它包含 两条线索: 顺序与互动。制作者可以预先准备一些卡片。 在每一张卡片上记录下旁白以及旁白所要表达的图像, 文 字以及文字所要传达的语言知识。一般来讲, 一段长度为 两分钟, 以语言教学为目标的“数字叙事”的故事板设计应 在 16 张左右, 每张图像的保持时间不低于 7 秒钟, 这样观 众有足够的时间来理解故事所传的意义和语言知识。

\section{2 运用电脑软件制作数字叙事}

用电脑自带程序或者其他录音设备将脚本录制成音 频。在音频录制时, 应根据英语学习者的水平来调整语流 的快慢以及停顿的频率。并通过语气、语调、重读来体现 重点内容。

利用网络资源选择静态或动态影像, 或者直接用数码 相机拍摄所需要的主题。根据教学目标所指定的语言内容, 选择表义贴切的影像, 并根据脚本、故事板进行修改, 调 整颜色对比度。

使用影像剪辑程序将音频资料和影像资料合成为整 体。将音频片段和视频片段按顺序导入, 根据教学内容调 整时间轴和声道的位置、持续时间, 突出教学重难点。以 PC 电脑自带程序 Moviemaker 为例: 打开程序后建立新项 目; 点击工具栏的收藏夹并命名; 将所有相关音频影像资
料导入收藏夹中, 以大图标显示; 根据教学内容和故事板 将音频和影像资料按顺序拖拽到窗口下方的工作区域, 调 整音轨和时间轴。

\section{3 电影故事的修改与发布}

“数字叙事”初步合成后, 可以根据教学需要适当加入背 景音乐, 背景音乐的声音应低于旁白的声音。Moviemaker 只有一个音轨, 音频混合则需要借助其他软件, 如 Audacity, 来实现。修改过程中, 给“数字叙事”添加效果、过渡以及 标题可能会延长画面预先所设定的时间, 这时应对影片各 部进行微调。

“数字叙事”修改之后, 便可导出作品, 发布并分享。 Moviemaker 能够轻松地实现整个发布过程: 将 movie 文件 夹保存后, 在菜单栏中选择发布方式（光盘方式或者网页 方式）后，将电影导出至指定文件夹即可。

\section{4 “数字叙事”的常用制作工具}

Windows Movie Maker。Windows Movie Maker 操作简 单, 容易掌握。Movie Maker 是 Windows 系统自带的视频 制作工具。在影音制作中, 可以执行以下操作：添加视频 和照片; 添加过渡特技、视觉效果、文本以及其他特殊修 饰; 添加片头、字幕。通过简单的拖放操作, 精心的篎选 画面, 然后添加一些效果、音乐和旁白, 数字叙事就初具 规模了。

iMovie 软件。iMovie 苹果机专用, 功能强大。iMovie 是一款由苹果电脑编写的视频剪辑软件。它允许用户剪辑 自己的家庭电影, 大多数的工作只需要简单地点击和拖拽 就能完成。它可以从大部分数码摄像机采集未经压缩数据, 直接输入 Mac 中。接着用 iMovie 剪辑、加入标题和音乐, 同时根据需要加入诸如淡入、淡出和幻灯等效果。

Adobe Premiere Elements 9。Adobe 公司推出的视频编 辑软件 Adobe Premiere Elements 9 能够提供自动化的影片制 作选项和专业品质的效果。它包含了能够从数码摄像机捕 捉视频的工具, 特效及过渡场景, 添加工具以及 DVD 刻录 功能。利用 Adobe Premiere Elements 9.0 软件只需两个简单 的步骤即可将画面刻录到 DVD 上, 也可尝试使用新的主题 电影制作模板来创建视频影像。

\section{4. 基于“数字叙事”的 ESL 课堂练习实例}

传统意义的原版视频外语教学是指在外语的视听说课 上使用原声影像资料进行听、说训练。“情景小电影”的外 语教学运用则是在此基础上的升华。除了呈现外语教学素 材, 它更加强调素材的选择和基于教学原理的创作性。利 
用数字化技术, “数字叙事” 将文本、影像、声音、动画特 效等多媒体素材综合加工成一个单独的个体来实施教学任 务。

\section{1 听力练习}

借助字幕将观众的注意力吸引到目标单词, 结构, 句 型和功能等语言点上, 借助停顿按钮, 让观众边听边写, 既可以采取完全听写的方式, 也可以采用完形填空的形式 听写摘要。采用听写的形式, 或者完形填空的形式。

实例: 数字叙事《What Do You Think About Weather Change?》

《你如何看待气候变化》为英语学习者练习四、六级 中的复合听写设计。

过程: 学习者一边观看一边记录。

Both the federal government and state government in the United States are working to For example, some laws require power plants and factories to reduce their carbon dioxide emissions, such as by using "scrubbers". Scrubbers are filtering systems that before they are released into the air. Certain newer systems can then store the carbon dioxide underground. Other laws require that

\section{2 会话练习}

角色扮演: 在观看完某个场景后, 让学生分角色重现 该场景。

现场采访: 播放完视频后, 让部分学生扮演记者, 就 场景中的某些细节随机采访其他学生, 然后进行现场新闻 报道。

信息配对: 将学生分为两组, 关掉一组学生的屏幕, 只保留声音; 关掉另一组的学生的耳麦, 只保留影像播放。 然后将两组的学生进行随机配对, 进行信息交流, 确定场 景, 情节, 人物, 观点等内容。

实例: 数字叙事 《Mayor Brown's Press Conference》

《布朗市长的记者招待会》为中、高级英语学习者练 习会话而设计。

过程: 学者边看边记录以下问题。

What is the problem with the streets?

How does the mayor respond?

What is the problem with the parks?

How does the mayor respond?

记录完毕后, 将学生分成两人或三人小组, 进行市长和记 者的角色扮演练习。

\section{3 阅读理解练习}

在播放前, 预先呈现某个场景中的一些关键词汇, 短 语或句子。在观看完对应场景后暂停播放, 让观众根据场 景描述对这些语言形式的理解, 并设计一系列的阅读练习 来 巩固观众对这些知识点的掌握。阅读练习可采用多项选 择, 判断正误, 信息配对, 信息填空, 顺序重组, 回忆特 殊句型等方式。

实例: 数字叙事《Signs Are Every Where》

《生活中的符号》为英语中级学习者练习功能性描述 而设计。

过程: 数字叙事播放完毕后, 先让学习者进行简单的 图文搭配练习, 回忆影片中的目标句型和词汇; 然后重播 并作静音处理, 要求学习者在没有任何提示的情况下, 用 目标语言描述各种符号的意义, 比如 these signs warn us about animals on the road, people make these signs for sales 等等之类的句型。

\section{4 写作练习}

摘要类写作: 让学生观看某个场景, 边看边记录其中 有用的语言信息; 观看结束后, 要求学生在规定时间内容, 根据所记录的信息写出一个段落的情节概要。

评论类写作: 学生就故事的内容写出他们感想, 态度, 发表评论。

创意类写作: 学生根据某个场景, 运用想象力, 构思 出故事发生的另一种可能, 并加以描述。

实例: 数字叙事 《Mayor Brown's Press Conference》

《布朗市长的记者招待会》也可以用于中、高级英语 学习者的写作练习。

过程: 播放完小电影后, 让学生写一篇新闻发布会的 现场报道。

\section{5. “数字叙事”模式运用于英语教学的意义}

以英语教学为目标的数字化讲述赋予语言学习特定的 情景, 提供丰富的语言输入量, 语言养成的视觉文化氛围, 帮助学习者形成有效的语言学习策略, 是语言学习者获得 语言的强有力工具。

\section{1 提供充有意义的语言输入}

20 世纪 80 年代, 美国语言学家克拉申[3]提出 “获得语 言的最佳方式是语言学习者在无焦虑状态下获得有意义的 语言输入”。数字化故事讲述中的图像, 音频和动画等多媒 体素材极大地丰富了语言信息输入量, 使语言的呈现更加 生动。同时多媒体的互动性也增强了学习者的学习兴趣和 
参与意识。语言学习者在故事所营造的真实语境中尝试各 种途径 (听、说、读、写) 来表达自己的理念或解决问题, 在语言的运用中获得语言。

以英语教学为目标的“数字叙事”应确保“有意义的语 言输入”。故事讲述的内容和意图应清晰明确; 对于比较生 僻或专业的内容应给与清晰的解释。例如, 3 分钟的数字叙 事《Blues: More Than A Feeling》(蓝调: 不仅仅是一种感 觉）向观众传达了蓝调音乐的概念, 发源, 所蕴含的音乐 元素, 音乐形式, 代表人物和作品以及对现代音乐的影响。 在讲述蓝调的音乐形式时, 小电影以动态图像形式展示了 大量的乐器, 并将经典蓝调作为其背景音乐。观众了解音 乐知识的过程中, 理解相关词汇, 术语或者复杂概念; 在 构建意义的过程中, 掌握词汇拼写和发音, 扩大词汇量, 提高语言运用能力。

\section{2 促成文化素养的生成和提高语言的流畅性}

通常意义的文化素养泛指处理文字的能力, 即对文字 的理解以及写作能力。高科技时代的文化素养含义则更为 广泛, 除了文字处理能力, 还涵盖对图像、音频的理解与 运用。以英语教学为目标的 “数字叙事” 将文字内容以故事 形式呈献给观众, 其中所蕴含的教学策略会引导他们以听、 说、读、写方式完成各种指定任务。在文化素养的生成中, 语言学习者的阅读、写作以及听说能力都能够得以提高。

引导英语学习者直接参与数字化故事创作是提高语言 流畅性的绝佳途径。例如以《校服》为话题, 要求学生组 成 2-5 人小组, 协作完成“数字叙事”的资料收集, 脚本创作, 录制, 剪辑, 合成与发布。数字化讲述的创作前期需要学 习者阅读大量原版文献资料, 在此基础上进行篮选、整合, 并融入自己的观点。中期的故事脚本制作则为学习者提供 了一个逼真的写作环境, 他们需要运用各种写作策略与技 巧来表述自己的观点。小电影的最终合成则是文字视觉化、 听觉化的过程, 对语言学者的听说能力都提出了较高的要 求。

\section{3 推动语言理解能力的纵深发展。}

“数字叙事”是以多媒体形式帮助观众构建意义的理解 过程。从认知学角度来讲, 理解过程涉及五个阶段: 微观, 连接, 宏观, 归纳、推理, 元认知[4]。微观是指对词义的
确认; 连接是指句子与句子, 段落与段落之间的关系; 宏 观是指整体构架; 推理是由信息得出结论; 元认知是对学 习策略的把握。五个阶段的结合推动理解向深层次发展。 以英语教学为目标的数字化讲述依靠内在的情节和技术要 件为观众提供深度理的各种策略。

“数字叙事”的情节要件包括：故事目的, 作者观点和 故事冲突。故事目的涉及 描述、说明、议论, 属于理解的 宏观阶段; 作者的观点包括支持/反对, 积极/消极等态度, 属于理解的推理阶段; 故事冲突涉及起因, 发展, 结局, 属于理解的宏观阶段。“数字叙事”的技术要件包括: 图像, 音频, 小标题和字幕。图像和音频都属于理解的微观阶段, 让观众直接感受故事所要表达的意义; 小标题属于理解的 连接阶段, 是整体结构。字幕的使用属于理解的微观阶段, 可以加深对语言形式的记忆。观众通过对情节以及技术要 件的把握, 加工、处理各种深层次信息, 从而推动逻辑思 维能力, 批判性思维能力向纵深发展。

“数字叙事”教学方式利于学习者在单位时间内有效地 掌握学习内容, 体会教学重难点, 形成有效的语言学习策 略。国外的一些研究显示: 高品质的多媒体讲述可以更好 地帮助学习者掌握词汇拼写和发音, 扩大词汇量, 提高逻 辑推理能力。语言学习者在故事情景所营造的文化素养氛 围中会尝试使用多种方式来表自己的理念和情感, 从而逐 步提高词语的分析能力, 阅读技巧以及阅读效果。以外语 教学为目标的“数字叙事”能够为语言学习者提供充足的、 有意义的语言输入与互动, 从视觉、听觉角度为语言学习 者营造一个身临其境的文化素养氛围, 在潜移默化中提高 语言学习者听、说、读、写综合能力。

\section{参考文献(References)}

[1] Center for Digital Storytelling, http://www.storycenter.org.

[2] B. R. Hergenhahn and M. H. Olson, An Introduction to Theories of Learning, Upper Saddle River, NJ: Prentice-Hall, 1997, pp.167-169.

[3] S.D.Krashen, Second Language Acquisition and Second Language Learning, Prentice-Hall International, 1988, pp. 89.

[4] E. Wenger, Communities of Practice. Learning, meaning and identity, Cambridge: Cambridge University Press,1999, pp.289-290. 